


\section{Le discours du pouvoir}

\section{BY: Bernard Lamizet}

\section{RÉSUMÉ}

T 1 importe de repenser, aujourd'hui, le discours du pouvoir, L en se fondant sur l'articulation sémiotique de quatre instances: l'énonciation politique, l'expression de l'identité de l'acteur dans son discours, la spécificité politique de la performativité du pouvoir et l'expression d'un inconscient politique dans le discours. Trois éléments définissent la spécificité de l'énonciation politique. Le premier est la définition d'enjeux propres à l'énonciation politique. Le second est la définition de ce que l'on peut appeler une écologie énonciative. Enfin, l'énonciation politique inscrit dans la communication des représentations des enjeux du pouvoir. L'énonciation du discours du pouvoir articule l'identité de l'énonciateur et un statut d'acteur politique. La communication politique se fonde sur la manifes-
INFO SUR L'ARTICLE:

Volume: 06

Issue: 02:2020

Month: March

ISSN: 2459-2943

DOI: 10.18680/hss.2020.0025

Pages: 141-154

Lic.: CC BY-NC-ND 4.0

MOTS-CLÉS:

énonciation politique

écologie énonciative

performativité

alientité tation d'une confrontation entre les acteurs dans l'espace de l'énonciation. La performativité politique se fonde sur l'identification de l'énonciation et de la manifestation d'un acteur politique. Cette performativité propre au politique se caractérise, dans le cas du discours du pouvoir, par deux faits: l'imposition des modalités de l'énonciation et de l'interprétation et, donc, de la communication, et l'imposition des enjeux de la communication, des références qui lui donnent sa consistance. Comme toute énonciation, l'énonciation du discours du pouvoir est l'expression d'un inconscient du pouvoir. On peut définir et analyser cet inconscient politique en se fondant sur ce que l'on peut appeler la connotation politique, qui définit une sémiotique du non-dit dans la communication politique. 


\section{Introduction}

Il importe de repenser, aujourd'hui, le discours du pouvoir, en se fondant sur l'articulation sémiotique de quatre instances: l'énonciation politique, l'expression de l'identité de l'acteur dans son discours, la spécificité politique de la performativité du pouvoir et l'expression d'un inconscient politique dans le discours. En effet, le discours du pouvoir présente trois spécificités qui peuvent faire l'objet d'une analyse sémiotique articulant ces quatre instances. D'abord, il met en scène une énonciation propre à un acteur à qui est reconnu un pouvoir, ce qui constitue une forme de performativité. C'est à la fois parce que la tenue du discours est mise en scène dans un espace institutionnel et parce qu'elle est portée par une voix à qui est reconnue un pouvoir que cette énonciation comporte une forme de performativité. Par ailleurs, l'énonciation du discours du pouvoir constitue, en soi, une manifestation de l'identité de l'acteur porteur du pouvoir dans l'espace public. En parlant en public, l'acteur qui tient le discours met en scène l'identité politique et l'engagement dont il est porteur et qui le situent dans l'espace politique. Enfin, comme tous les discours politiques, mais sans doute d'une façon qui lui est propre, le discours du pouvoir s'articule à un inconscient. Il existe une forme inconsciente du discours politique, fondée à la fois sur les connotations de ce discours qui renvoient à ce que l'on peut appeler un inconscient politique partagé, commun à l'énonciateur et à ceux qui écoutent son discours ou qui le lisent, et à des traces de formes particulières de refoulement politique, comme on le verra dans l'analyse du discours d'E. Macron étudié ici.

C'est que nous n'avons pas choisi de parler du discours du pouvoir de façon générale, sans nous fonder sur l'analyse d'un discours particulier, mais que nous avons donné à notre propos l'exemple d'un discours prononcé par le président de la République devant des élus, ${ }^{1} c^{\prime}$ est-à-dire devant d'autres acteurs politiques porteurs de pouvoirs particuliers. C'est ainsi que le discours d'E. Macron offre l'intérêt de permettre $\mathrm{d}^{\prime}$ analyse ce que l'on peut appeler la mise en scène performative de la confrontation de pouvoirs différents dans l'espace politique. Au-delà, on peut même dire que ce discours constitue une illustration de ce que devient l'espace politique dès lors qu'il est ainsi mis en scène par un discours. En effet, le propre du discours politique est d'engager ce que l'on peut appeler la performativité du discours de l'autre. Au cours des recherches que nous avons menées sur le discours politique, nous étions parvenu à la définition d'une particularité du discours politique, qui le distingue d'autres types de discours et qui fonde la singularité de son énonciation: le discours politique engage la performativité du discours de l'autre. Plus précisément, le propre du langage politique est

\footnotetext{
1 Prononcé devant le congrès des maires et des présidents d'intercommunalités, le 19 novembre 2019, ce discours peut être lu in extenso grâce au lien suivant qui permet de se connecteur au site de la présidence de la République: https:/ / bit.ly/3dmbvPs
} 
que son métalangage est l'action de l'autre, ou son engagement dans l'espace politique, ce qui caractérise l'action politique. C'est l'énonciation du discours politique qui définit l'identité de l'acteur politique en situant son engagement par rapport à lui, mais c'est parce qu'il se confronte à un adversaire, ou à un autre acteur de l'espace public que son énonciation revêt un caractère pleinement politique. C'est ainsi que, dans cette perspective, le propre du discours du pouvoir sera d'engager la performativité de ceux qui l'écoutent ou de ceux qui le lisent en suscitant l'expression de leur opinion ou de leur engagement. Dans le cas du discours d'E. Macron analysé ici, ceux à qui il est destiné, détenteurs de pouvoirs locaux, participent à ce que l'on peut appeler la rencontre de leurs pouvoirs avec le pouvoir du président de la République, dans une forme de confrontation que l'on peut définir comme une forme de spécularité des pouvoirs. C'est cette spécularité même qui permet de penser ce que l'on peut appeler la sémiotique de l'énonciation du discours du pouvoir. Cette spécularité des pouvoirs se distinguent de la spécularité psychique par le fait qu'elle ne se fonde pas sur l'identification spéculaire de l'énonciateur à l'autre, mais, au contraire, sur leur confrontation, sur l'adversité qui les situe dans l'espace politique.

\section{L'énonciation politique}

Trois éléments définissent la spécificité de l'énonciation politique. Le premier est la définition, dans le discours, d'enjeux propres à l'énonciation politique. En effet, tandis que l'énonciation ordinaire peut ne pas se situer par rapport à des enjeux, l'énonciation politique manifeste toujours des enjeux du discours, qui sont la forme énonciative des enjeux de la confrontation autour du pouvoir. C'est ainsi que, dans le discours d'E. Macron que nous analysons ici, on peut relever trois enjeux propres. Il s'agit, d'abord, de l'égalité, qui est figurée, dans les propos d'E. Macron, par la nécessité d'unir et rassembler et par un nouvel aménagement de notre territoire. On relèvera, d'ailleurs, que, dans ce discours, c'est le nous qui représente l'identité de l'énonciateur, ce qui est une manière de refouler les oppositions, les confrontations, ce qu'il appelle les fractures, en disant, un peu plus haut: "fractures territoriales, fracture numérique, sociale, identitaire, culturelle aussi, trop de fractures, comme si la France n'était plus une." En refoulant, sous la référence à ce 'nous,' les différences et les divergences qui font d'eux des acteurs politiques, E. Macron confond les identités politiques des personnalités du débat public en les faisant porteurs d'une forme artificielle de communion, comme dans le discours de l'Église.

La seconde caractéristique de l'énonciation du discours politique est de définir ce que l'on peut appeler une écologie énonciative, c'est-à-dire un ancrage du discours dans l'espace politique et dans l'espace public du débat. "Le temps," dit E. Macron, "est à la redéfinition d'un espace et d'un commun." C'est de cette manière que ce que nous nous proposons de dessiner par le concept d'écologie énonciative s'élabore, au fur et à me- 
sure de l'énonciation du discours, le discours politique engageant la construction de cette écologie, de cet espace-temps propre à l'énonciation. Mais la figure 'd'un commun' va plus loin: en effet, il s'agit bien de définir des modalités de l'énonciation reconnues par l'ensemble des partenaires de la communication, c'est-à-dire de ceux qui participent à la communication engagée par l'énonciation. Si nous proposons de reconnaître cette écologie énonciative comme une caractéristique du discours politique, c'est que l'une des significations majeures de l'énonciation qu'il met en œuvre est ce que l'on peut appeler l'institution d'un espace-temps, c'est-à-dire d'une écologie partagée par l'ensemble de ceux qui participent à la médiation et à la communication politique. Mais cette figure du 'commun' est très précisément ce que représente le terme oikos du concept d'écologie, l'oikos désignant, en grec, l'ensemble de ce qui constitue l'espace commun, qu'il s'agisse de l'espace privé familial ou de l'espace connu, parcouru et habité par l'ensemble de ceux qui sont porteurs de la même identité.

Enfin, l'énonciation politique définit des références du pouvoir, elle inscrit dans la communication des représentations des enjeux du pouvoir, partagés entre l'énonciateur et ceux qui le lisent ou l'écoutent. Dans le discours d'E. Macron, on peut ainsi relever cinq références par rapport auxquelles se situe l'énonciation. Il s'agit de la fiscalité à laquelle se réfère le discours pour proposer d'en élaborer de nouvelles règles (p. 1), de l'union et du rassemblement, qu'il propose de mettre en œuvre par l'engagement de "rituels," qui sont des représentations institutionnels d'une identité partagée (p. 2 et 4), d'actes, définis (p. 3) comme "ce travail quotidien résolu pour faire en sorte que chaque terre de France soit une chance pour celles et ceux qui y vivent, par plus de stages proposés, par les emplois francs développés." Par ailleurs, il s'agit de la sécurité (sécurité, vigilance, police apparaissent dans le discours, p. 4) et, enfin, il s'agit de la figure de la laïcité (p. 5), qui occupe une place particulière dans le discours politique français depuis que sont apparues les tensions liées aux manifestations des radicalismes religieux. Ce sont ces enjeux de l'exercice du pouvoir, ici présentés et définis par E. Macron, qui définissent pleinement les modalités de l'exercice du pouvoir et le concept même de souveraineté dans une société démocratique.

C'est de cette manière que l'énonciation politique définit une écologie énonciative, qui fonde la spécificité d'une rhétorique et d'un lexique construisant des logiques énonciatives partagées entre l'énonciateur et son public. En effet, l'énonciation politique se caractérise par l'institution d'une situation de communication associant l'énonciateur et les autres acteurs de la communication dans la représentation partagée de l'espace et $\mathrm{du}$ temps reconnus comme ce qui fonde le champ politique, ce que l'on peut appeler l'agora, c'est-à-dire un espace d'information, de débat et d'échanges symboliques habité (c'est le sens de oikos et du terme écologie) par des acteurs de communication partageant leur identité par le fait de participer à la même situation de communication. L'écologie énonciative dans laquelle s'inscrit le discours du pouvoir ne se limite pas, bien sûr, au 
discours des acteurs du pouvoir, mais elle constitue fondamentalement un espace d'énonciation du discours politique.

\section{L'expression discursive de l'identité de l'acteur}

La communication politique se fonde sur l'identification de ses énonciateurs, de ceux qui énoncent le discours politique, c'est-à-dire d'acteurs politiques. Cela signifie qu'elle repose sur la reconnaissance de l'identité politique et de l'engagement dont ils sont les acteurs par ceux qui parlent, mais aussi par ceux à qui ils s'adressent. En effet, l'identité des énonciateurs du discours politique se fonde toujours sur l'identification entre des subjectivités et des acteurs. Tandis que, dans les lieux et les circonstances ordinaires de la communication, l'énonciateur du discours et de la parole est un sujet, dont l'identité symbolique, en particulier, se fonde sur une relation spéculaire d'identification symbolique à l'autre sujet à qui il parle, à qui il s'adresse, avec qui il engage un échange de paroles, dans l'énonciation du discours politique, l'identité de l'énonciateur se fonde sur la confrontation à l'autre, qu'il s'adresse à lui ou qu'il le mentionne dans son discours. Le discours politique ne se fonde pas sur l'identification spéculaire de l'énonciateur à l'autre, mais sur sa confrontation à lui. C'est ainsi, par exemple, que, dans le discours qui nous occupe, il s'agit d'une confrontation entre un acteur porteur d'un pouvoir, le président de la République, et d'autres acteurs, porteurs d'autres pouvoirs, les élus municipaux. Dans ce champ, qui n'est pas un espace spéculaire d'échange symbolique, mais l'espace public, lieu de mise en œuvre de la confrontation entre des identités politiques, l'énonciation du discours du pouvoir articule l'identité de l'énonciation et un statut d'acteur du pouvoir, figure à la fois définie par le processus de l'énonciation et par la reconnaissance dont elle fait l'objet de la part de ceux à qui le discours est destiné. Tandis que la communication se fonde toujours sur l'identification spéculaire des énonciateurs qui participent à l'échange symbolique, ce qui est la raison pour laquelle elle engage des sujets, ${ }^{2}$ la communication politique se fonde, de façon spécifique, sur la manifestation d'une confrontation entre les acteurs dans l'espace de l'énonciation. On ne peut s'identifier symboliquement à un acteur porteur d'un pouvoir, car s'il le détient, c'est que nous ne le détenons pas, et on ne peut s'identifier symboliquement à un adversaire politique, car ce qui fonde notre identité est, précisément, ce qui situe la différence entre nos engagements.

C'est cette confrontation qui est évoquée, par exemple, dans le discours d'E. Macron, par ces mots: "les mots n'épuisent pas ce que doit être notre réaction collective face au regain des passions, de la division et de la haine" (p. 4), ou encore (p. 5): "bien souvent, ce qui

\footnotetext{
${ }^{2}$ Le terme sujet est issu, étymologiquement, du latin subjectum, c'est-à-dire situé sous le regard de l'autre.
} 
traverse notre société, ce sont les sujets que je viens d'évoquer, légitimes, qui touchent la civilité, l'égalité entre les femmes et les hommes, le respect des lois de la République, l'ordre public, et c'est là qu'il nous faut collectivement nous réarmer, peut-être prendre des lois nouvelles, des décisions plus fortes." Si, dans son discours, E. Macron emploie de tels mots qui se réfèrent à la violence et à la guerre, en particulier dans la figure du 'réarmement,' c'est bien que le président de la République entend, en quelque sorte, profiter de ce discours pour rappeler une fois de plus qu'il se fonde sur sa confrontation aux autres acteurs politiques de l'espace public.

Mais il faut aller plus loin. Dans son discours, E. Macron soulève plus précisément la question de l'identification des acteurs politiques, en particulier quand (p. 5) il dit: "Ce qui compte, c'est de faire tomber les masques de ceux qui combattent la devise inscrite sur les frontons des mairies qu'ils convoitent." Le discours du président s'inscrit ainsi dans la logique de ce que l'on eut appeler l'élucidation des identités, dans le projet de la mise en œuvre d'un éclairage de l'identité des acteurs engagés dans la confrontation qui donne ce que l'on peut appeler son actualité à l'espace public. C'est la signification qu'il convient de donner à l'expression de ce qu'il appelle le 'solidarisme' (p. 7): "la République sociale, solidaire," "ce solidarisme contemporain auquel je crois profondément." La figure de la solidarité avait été introduite dans l'espace politique français par F. Mitterrand, quand, en 1981, il avait, pour la première fois, institué, dans notre pays, un "ministère de la Solidarité." Il s'agissait, alors, d'unifier l'ensemble des politiques de protection sociale, et cette figure de la solidarité a été, en quelque sorte, amplifiée, aujourd'hui, rendue plus urgente, par la montée du chômage et des suppressions d'emplois, et par la survenue de la pandémie du coronavirus.

C'est, enfin, dans cette logique de la confrontation, en situant ses auditeurs dans le même champ que lui, que le président va évoquer ce qui fait d'eux des acteurs: "Votre moteur," dit-il (p. 9), "c'est de faire, de transformer, d'agir." De cette manière, le discours d'E. Macron inscrit la communication politique dans une logique de l'acte et non dans une logique de la parole: il ne s'agit pas seulement de mots ou de discours, mais il s'agit bien d'engager des actions dans l'espace politique. C'est, d'ailleurs, bien la raison pour laquelle, dans son discours, E. Macron va parler des actes à venir des maires à qui il s'adresse, en évoquant les "défis" (p. 6) à relever: "Pour réussir ces transformations," ditil (p. 6), "comme à chaque fois que la République a été confrontée à ces grandes bourrasques, j'ai besoin de vous, et c'est ensemble que nous battrons cette action utile." En fondant l'identité de ses auditeurs sur une logique de l'action, le président de la République à la fois situe le discours politique dans une rhétorique performative et reconnaît à ceux à qui il parle un pouvoir, fondé sur l'action, qui, précisément parce qu'il ne se fonde que sur de l'acte, et non sur la réalité de la reconnaissance d'un pouvoir, a de fortes tendances artificielles, voire illusoires. 


\section{La performativité du discours du pouvoir}

Le discours des acteurs du pouvoir s'inscrit dans une logique performative, car, par le discours qu'il tient dans l'espace public, le pouvoir se manifeste, met en scène une forme de réalité de l'exercice du pouvoir. Mais il faut aller plus loin: en réalité, ce n'est pas en propre le discours du pouvoir qui engage une performativité: c'est le méta-langage du discours du pouvoir qui s'inscrit dans la logique de l'acte. En-dehors de quelques situations particulières, que nous allons étudier ici, le discours du pouvoir n'est pas en soi un performatif, ce qui engage de l'action, c'est l'interprétation de ce discours par les acteurs à qui il s'adresse.

Commençons donc par analyser les situations institutionnelles au cours desquelles les acteurs du pouvoir engagent une forme de performativité de leur discours, de leur parole. On donnera trois exemples de ces situations. Le premier exemple que l'on peut donner est classique: il s'agit de la performativité des discours que l'on peut appeler les discours institutionnels. Quand un président de séance, porteur d'un pouvoir, dit "La séance est ouverte," cela suffit à ce que la séance ait lieu. C'est aussi le cas des formules par lesquelles le président de la République promulgue une loi. Quand ces mots figurent en tête d'une loi: "L'Assemblée nationale et le Sénat ont adopté, le président de la République promulgue la loi dont la teneur suit," cela suffit à imposer l'application de la loi. Un second exemple est courant, même s'il n'est pas toujours conscient. Quand, à la même question posée par le maire, successivement, à l'un et à l'autre: "Acceptez-vous de prendre pour époux ... ?," une femme et un homme répondent par un simple "oui," cela suffit à ce que l'un et l'autre soient mariés. Enfin, on peut donner un troisième exemple dans les situations de crise, au cours desquelles les pouvoirs exécutifs mettent en œuvre un ensemble de mesures destinées à faire face à cette crise, comme c'est le cas, aujourd'hui, dans la situation de crise engagée dans de nombreux pays par la pandémie du coronavirus, en mars 2020. Sans doute même faut-il aller plus loin, et définir le concept même de pouvoir exécutif comme le pouvoir à qui est reconnue la compétence d'imposer des mesures destinées à assurer ce que l'on peut appeler la permanence de l'État, sa pérennité, sa sécurité. Ce que Montesquieu désigne par le pouvoir exécutif n'est pas seulement le pouvoir d'appliquer et de mettre en œuvre les lois votées par le pouvoir législatif, mais il s'agit du pouvoir d'engager la performativité méta-langagière qui se met en œuvre dans une situation politique et institutionnelle particulière. Si le pouvoir a une réalité, c'est que le méta-langage qui lui donne sa signification relève de l'acte et non d'une simple réponse ou d'un autre discours.

Mais il faut aller plus loin dans cette analyse de la performativité du discours du pouvoir. Tandis que le performatif se fonde toujours sur l'identification d'une parole et d'un acte, la performativité politique se fonde sur l'identification de l'énonciation et de la manifestation d'un acteur politique. Ce n'est pas la mise en œuvre d'un simple 
acte qui définit la performativité du discours du pouvoir, c'est l'institution d'une situation politique particulière, c'est l'imposition d'une contrainte nouvelle à l'ensemble de la population d'un pays sur lequel règne l'exécutif. Ce qui définit l'identité énonciative d'un acteur politique, c'est que l'acte articulé à la performativité de son discours s'inscrit dans une suite d'actions et d'engagements qui l'ont précédé et qui ont pu faire évoluer les enjeux des discours du pouvoir ou les références de sa confrontation aux autres discours politiques de l'espace public. La performativité du discours du pouvoir s'inscrit ainsi dans une mémoire des médias et des autres acteurs politiques et dans une histoire qu'elle définit.

La performativité propre au politique se caractérise, dans le cas du discours du pouvoir, par deux faits : le premier est l'imposition des modalités de l'énonciation et de l'interprétation et, donc, de la communication, et l'autre est l'imposition des enjeux de la communication, des références qui lui donnent sa consistance.

Imposer les modalités de l'énonciation et de la communication constitue une contrainte imposée par les acteurs porteurs du pouvoir. C'est ainsi que (p. 7) E. Macron entend imposer sa conception des mobilités et des logiques qui les structurent. "Je crois," dit-il, ainsi, "à une écologie de la liberté, de l'innovation. Je crois dans l'écologie des territoires et la capacité que l'on donne à ces derniers de trouver les bonnes solutions." D'abord, il convient de relever, dans ces mots, la répétition du terme "Je crois," qui inscrit le discours du chef de l'État dans une logique de la croyance, ce qui engage une diminution de la critique appelée par ces propos. Mais, surtout, dans la conception de l'écologie qui est affichée par E. Macron à propos des transports et des déplacements, ce qu'il importe de noter est l'articulation de trois éléments qui donne une pleine signification à ce discours et permet de mieux le comprendre. Il s'agit de l'articulation entre écologie, innovation et liberté, c'est-à-dire entre trois éléments qui orientent ce discours: l'écologie, c'est l'orientation de l'aménagement de l'espace qui entend en conserver ce qui le fonde comme espace d'habitation et de protection de l'environnement contre les dégradations qu'il pourrait subir; l'innovation, c'est l'expression d'une référence au mythe de la modernité, qui fait partie des orientations de la politique industrielle; la liberté, enfin, c'est la référence, en quelque sorte obligatoire, au libéralisme, qui est une orientation fondamentale de la politique économique élaborée et mise en œuvre par E. Macron depuis son accession au pouvoir.

Mais imposer les modalités de la communication signifie aussi imposer des contraintes de l'interprétation du discours et des significations que l'on est amené à lui donner. C'est dans cette logique qu'E. Macron fait référence, à la fin de son discours ( $\mathrm{p}$. 12) à la logique de l'engagement. "Ce qui compte," dit-il, "c'est l'engagement. Ce qui compte, c'est que la République demeure vive." Dans cette référence, à la conclusion de son propos, à l'engagement et à sa nécessité politique, on peut trouver un lien avec le "je crois" évoqué plus haut. Il s'agit, en réalité, d'une articulation entre la dimension singulière de 
l'acteur et son inscription dans l'espace public. Il y a, dans cette référence à l'engagement et à ce qu'E. Macron appelle sa nécessité en disant que c'est lui qui "compte," une référence à l'articulation de l'identité de l'acteur politique entre sa personnalité singulière et la reconnaissance dont il fait l'objet dans la dimension collective de son inscription dans l'espace public. Finalement, en évoquant cette nécessité de l'engagement, E. Macron situe son discours dans une sorte d'histoire des acteurs politiques, mais, surtout, dans une forme de dimension morale, éthique, des pratiques sociales qu'ils mettent en œuvre. C'est par l'engagement dont ils sont porteurs que les acteurs politiques - en particulier ceux à qui s'adresse E. Macron dans son discours - fonde leur légitimité et la légitimité de leur pouvoir.

Les acteurs porteurs du pouvoir imposent à la situation de communication qu'ils dominent les enjeux de la parole et du discours, ainsi que les références qui fondent la signification du discours. Détenir un pouvoir et se le faire reconnaître par les autres acteurs de l'espace politique, ce n'est pas seulement engager sa représentation du pouvoir et en manifester la réalité dans l'espace public, c'est aussi déterminer les 'règles du jeu' qui définissent les formes et les structures de l'espace politique du discours et de l'énonciation. C'est bien pourquoi le pouvoir politique est à la fois un pouvoir réel - celui de l'action et de la décision, un pouvoir symbolique - celui qui consiste à instituer les règles de l'énonciation à un moment donné de l'histoire du discours politique, et un pouvoir imaginaire - car, si l'on détient un pouvoir, c'est aussi parce que ceux à qui on s'adresse dans les médias ou dans la réalité de l'espace public, se font une certaine représentation du pouvoir que l'on détient dans l'imaginaire politique dont ils sont porteurs.

\section{Un inconscient politique}

Si nous situons l'énonciation du discours politique dans l'articulation d'une instance réelle, d'une instance symbolique et d'une instance imaginaire, c'est en nous référant aux travaux du psychanalyste J. Lacan, qui définit ces trois instances comme ce que l'on peut appeler les trois lieux de l'espace du psychisme et de l'identité du sujet. Comme toute énonciation, l'énonciation du discours du pouvoir est l'expression d'un inconscient du pouvoir, d'un ensemble d'instances faisant l'objet d'un refoulement qui rend la communication possible en définissant des lois imposées aux énonciateurs et aux autres participants à la communication politique. On peut définir et analyser cet inconscient politique en se fondant sur ce que l'on peut appeler la connotation politique, qui définit une sémiotique du non-dit dans la communication politique.

C'est ainsi, d'abord, qu'il convient de comprendre l'expression par E. Macron d'un souhait de déconcentration. "Le numérique dont on parle tant," dit-il, "auquel nous tenons, qui nous permet de recréer de l'industrie, de développer des emplois sur notre territoire, doit 
nous permettre aussi d'être inventifs en matière d'organisation de l'État." Ce propos s'accompagne, dans les propos d'E. Macron, d'une critique des modalités de la décentralisation mise en œuvre jusqu'à présent. "Il faut," dit-il, "que la décentralisation s'accompagne de choix clairs du côté de l'État, il doit lâcher toutes les compétences quand il les décentralise, mais il ne faut pas que ça conduise à la perte collective de connaissances et que, d'un seul coup, on se réveille et qu'on se dise: là, on n'est plus aussi bons qu'avant." C'est ainsi que, dans son propos, E. Macron met en œuvre une critique de la façon dont l'État a mis en œuvre, jusqu'à présent, les processus de la décentralisation et de la déconcentration. Il s'agit bien, dans son discours, d'une forme de critique de l'État et de ce qu'E. Macron appelle l'absence d'une "fiscalité claire."

Cette critique de la politique fiscale élaborée et mise en œuvre en France est désignée par le président de la République comme un "fétichisme français." "Nous, nous avons un fétichisme français: l'autonomie fiscale," dit E. Macron, en poursuivant ainsi son propos: "Je regarde les grands pays décentralisés autour de nous, ils sont beaucoup plus décentralisés que nous, ils n'ont pas d'autonomie fiscale." "Ils ont une chambre," poursuit-il, "qui, chaque année, en effet, discute des ressources fiscales qui sont affectées aux collectivités, chaque niveau avec des règles claires. Peut-être qu'il faut en arriver à cela, et, moi, j'y suis favorable parce que, je vais vous dire, l'autonomie fiscale a deux problèmes." Et le président poursuit en évoquant ces deux problèmes, le premier est le fait qu'" il n'y aura jamais la bonne fiscalité, en tout cas pour les départements et les régions," et le deuxième est ce qu'il appelle "l'illisibilité" de la fiscalité locale. C'est ainsi que, dans cet inconscient politique qu'il présente dans son propos, E. Macron évoque, au sujet de la fiscalité locale, ce qu'il appelle "un fétichisme français," à propos de "l'autonomie fiscale."

C'est, d'ailleurs, dans cette logique de la critique de l'autonomie fiscale des collectivités locales qu'E. Macron évoque, dans son discours, une culture des cahiers de doléances. "J'ai, en ce moment," dit-il à la fin de son discours, "une pensée et un remerciement particulier pour Vanik Bernerian qui, avec ces maires, est venu me présenter ses premiers cahiers qu'il avait ouverts en mairie." En évoquant ces initiatives de certains élus locaux, notamment à l'occasion du conflit des 'gilets jaunes,' E. Macron parle de maires qui ont "proposé et innové," et il définit ainsi le rôle des élus: "de la commune à la nation," dit-il, "tel est notre rôle: rappeler les droits et les devoirs à chacun, ne jamais oublier cet 'en même temps' républicain auquel je crois profondément." Dans son discours aux maires et aux présidents des collectivités locales, le président de la République définit "la République" comme "cette soif d'agir," "ces mille fils tendus, cette amitié profonde que nous devons rappeler à nos concitoyens," c'est-à-dire comme un espace politique sans conflits et sans tensions sociales.

Sans doute, d'ailleurs, cette absence de conflit s'inscrit-elle, dans le propos d'E. Macron, dans la logique d'une sorte de refoulement. Les gens, dit-il, "veulent prendre des compétences et pas les responsabilités." "On ne peut pas avancer comme ça," poursuit-il, "Donc oui à un grand débat, une grande avancée sur la décentralisation, mais à condition de dire que 
la compétence va avec la responsabilité démocratique et claire, avec des financements clairs, qui ont la même dynamique, et là, nous serons heureux." Sans doute, dans le propos du président, est-ce cette forme de refoulement qui rend possible l'institution d'une véritable démocratie reposant sur une fiscalité locale rationnelle et acceptée.

\section{Pour conclure}

Quatre éléments semblent essentiels, dans cette approche de la performativité du discours politique.

Le premier est que, finalement, on peut dire qu'il n'y a de discours pleinement politique que dans cette performativité du pouvoir ou de l'opposition au pouvoir. De la même manière que Lacan parle de parole pleine pour désigner une parole qui énonce le désir dont est porteur le sujet, on peut parler d'une parole politique pleine pour désigner un discours dont la rhétorique ne consiste pas seulement à séduire ou à convaincre, mais consiste à faire reconnaître par l'autre le pouvoir dont on est porteur. En ce sens, chaque énonciation d'un discours par un pouvoir est une remise en question de cette plénitude de la parole dans ce que l'on peut appeler l'événement de la tenue du discours et de sa rencontre avec les autres dans l'espace public.

Le second élément important qui caractérise la performativité du discours politique est qu'elle se situe dans l'espace et dans le temps qui la définissent et lui donnent sa signification. Sans doute, d'ailleurs, est-ce là la limite majeure du discours politique: hors de l'espace politique, du pays, dans lequel il est tenu et à qui il s'adresse et en-dehors de la période pendant laquelle celui qui parle est porteur du pouvoir, le discours du pouvoir n'a plus de signification. Tout juste peut-il se voir reconnaître la valeur d'un témoignage ou d'une référence de l'histoire politique.

Par ailleurs, le discours politique est pleinement performatif parce que les mots mêmes qu'il emploie sont chargés de connotations institutionnelles qui donnent son caractère politique à leur signification. Le lexique politique lui-même revêt un caractère performatif : ce n'est pas seulement l'énonciation du discours et la parole elle-même qui sont porteuses d'une performativité, mais cette performativité même se situe dans les mots et dans la signification que lui donnent ceux à qui ils s'adressent.

Enfin, comme son énonciation se fonde sur la confrontation de celui qui parle et de l'autre, le discours politique, il s'inscrit dans une problématique particulière de l'identité, celle que nous avons définie comme l'alientité, qui se fonde sur la différence avec l'autre au lieu de se fonder sur l'identification à lui. C'est cette logique de l'alientité qui définit l'espace public comme un espace pleinement politique, comme un espace dans lequel les pouvoirs et les acteurs politiques s'opposent les uns aux autres dans la tenue de leur discours et dans la mise en scène de leurs manifestations dans l'espace public. 


\section{Bibliographie géneral}

Arendt, Hannah 1995. Qu'est-ce que la politique? Texte établi par U. Ludz et traduit par S. Courtine-Denamy. Paris: Seuil.

Arendt, Hannah 1972. Le système totalitaire. Tr. par J.-L. Bourget, R. Dvreu et P. Lévy. Paris: Seuil.

Aristote 1968-1971. Politique, 3 vol. Tr. par J. Aubonnet. Paris: Les Belles Lettres.

Aristote 1991-2000. Rhétorique, 3 vol. Tr. par M. Dufour. Paris: Les Belles Lettres.

Austin, John L. 1970. Quand dire, c'est faire. Tr. par G. Lane. Paris: Seuil.

Baudrillard, Jean 1972. Pour une critique de l'économie politique du signe. Paris: Gallimard.

Benveniste, Emile 1971. Problèmes de linguistique générale. Paris: Gallimard.

Benveniste, Emile 1980. Problèmes de Linguistique générale II. Paris: Gallimard.

Blondiaux, Loïc 1998. La fabrique de l'opinion. Paris: Seuil.

Bonnafous, Simone, Pierre Chiron, Dominique Ducard et Carlos Levy (dir) 2003. Argumentation et discours politique. Antiquité grecque et latine, Révolution française, monde contemporain. Rennes: Presses Universitaires de Rennes.

Breton, Philippe 2000. La parole manipulée. Paris: La Découverte.

Breton, Philippe 1997. L'utopie de la communication. Paris: La Découverte.

Breton, Philippe 1996. L'argumentation dans la communication Paris: La Découverte.

Cabasino, Francesca 2001. Formes et enjeux du débat public. Discours parlementaire et immigration. Roma: Bulzoni.

Cassirer, Ernst 1993. Le mythe de l'État. Tr. par B. Vergely. Paris: Gallimard.

Cayrol, Roland 1997. Médias et démocratie. La dérive. Paris: Presses de Sciences Po.

de Certeau, Michel 1968. La prise de parole. Paris: Desclée de Brouwer.

Charaudeau, Patrick et al. 2005. Le discours politique. Paris: Vuibert.

Chebel, Malek 1998. La formation de l'identité politique. Paris: Payot.

Clément, Catherine 1973. Le pouvoir des mots. Paris: Mame.

Colas, Dominique 2006. Sociologie politique. Paris: P.U.F.

Cordellier, Serge (dir.) 2000. Dictionnaire historique et géopolitique du vingtième siècle. Paris: La Découverte et Syros.

Cubertafond, Alain 1993. Le pouvoir, la politique et l'État en France. Paris: Livre de Poche, Deleuze, Gilles 1969. Logique du sens. Paris: U.G.E.

Derrida, Jacques 1979[1967]. L'Écriture et la différence. Paris: Seuil.

Descamps, Jean-Luc et al. 1992. Sémantique et concordances, suivi du Dictionnaire contextuel de français politique. Paris: Klincksieck.

Didier-Weill, Alain 1995. Les trois temps de la loi. Paris: Seuil.

Dragan, Ion (dir.) 1999. La communication du politique. Paris: L'Harmattan.

Duhamel, Alain 1995. La politique imaginaire. Paris: Gallimard. 
Foucault, Michel 1966. Les mots et les choses. Paris: Gallimard.

Friedberg, Erhard 1993. Le pouvoir et la règle. Paris, Seuil.

Gauchet, Marcel 2005. La condition politique. Paris: Gallimard.

Gerstlé, Jacques 1993. La communication politique. Paris: P.U.F.

Habermas, Jürgen 1993 [1962]. L'espace public. Tr. par M. B. de Launay. Paris: Payot.

Habermas, Jürgen 2000. Après l'État-nation. Une nouvelle constellation politique. Tr. par R. Rochlitz. Paris: Fayard.

Hobbes, Thomas 1999. Léviathan. Tr. par F. Tricaud. Paris: Dalloz.

Kapferer, Jean-Noël 1995. Rumeurs. Paris: Seuil.

Lacan, Jacques 1966[1949]. Le stade du miroir comme formateur de la fonction du Je, telle qu'elle nous est révélée dans l'expérience psychanalytique. Écrits. Paris: Seuil, 93-101.

Laclau, Ernesto 2000. La guerre des identités. Tr. fr. par C. Orsoni. Paris: La Découverte.

Lamizet, Bernard 1992. Les lieux de la communication. Liège: Mardaga.

Lamizet, Bernard 1998. La médiation politique. Paris: L'Harmattan.

Lamizet, Bernard 2002. Politique et identité. Lyon: Presses Universitaires de Lyon.

Lamizet, Bernard 2011. Le langage politique : discours, images, pratiques. Paris: Ellipses.

Lamizet, Bernard 2015. L'Alientité. Pais: Mimésis.

Lamizet, Bernard 2016. Communication et médiation. Saarbrücken: Éditions universitaires européennes.

Laplantine, François 1999. Je, nous et les autres. Paris: Le Pommier-Fayard.

Lefebvre, Henri 1966. Le langage et la société. Paris: Gallimard.

Lefort, Claude 1986. Essais sur le politique: XIXème-XXème siècles. Paris: Seuil.

Legendre, Pierre 2001. De la Société comme Texte. Paris: Fayard.

Legendre, Pierre 1974. L'amour du censeur. Paris: Seuil.

Marin, Louis 1981. Le portrait du roi. Paris: Minuit.

Marx, Karl et Friedrich Engels 1970. L'idéologie allemande. Paris: Éditions Sociales.

Mesure, Sylvie et Alain Renaut 1999. Alter ego. Les paradoxes de l'identité démocratique.

Paris: Aubier.

Negri, Antonio 1997. Le pouvoir constituant. Tr. fr. par É. Balibar et F. Matheron. Paris: P.U.F.

Ramonet, Ignacio 2000. Propagandes silencieuses. Paris: Galilée.

Sfez, Lucien 1988. Critique de la communication. Paris: Seuil.

Tchakhotine, Serge 1992[1952]. Le viol des foules par la propagande politique. Paris: Gallimard.

Tournier, Maurice 1997. Des mots en politique. Propos d'étymologie sociale, vol. 2. Paris: Klincksieck. 
Weber, Max 1963. Le savant et le politique. Tr. par J. Freund. Paris: U.G.E.

Wolton, Dominique 1991. Les médias, maillons faibles de la communication politique.

Hermès 4: 165-178.

Wolton, Dominique 1997. Penser la communication. Paris: Flammarion.

Zémor, Pierre 1999. La communication publique. Paris: P.U.F.

\section{AUTEUR}

Bernard Lamizet est Professeur de Sciences de l'information et de la communication, Institut d'Études Politiques de Lyon, France. 


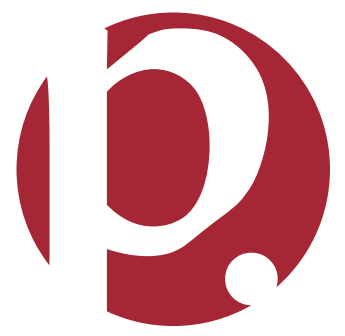

